\title{
ON SOME SUFFICIENT CONDITIONS FOR THE BLOW-UP SOLUTIONS OF THE NONLINEAR GINZBURG-LANDAU-SCHRÖDINGER EVOLUTION EQUATION
}

\author{
SH. M. NASIBOV
}

Received 8 March 2003 and in revised form 1 November 2003

Investigation of the blow-up solutions of the problem in finite time of the first mixedvalue problem with a homogeneous boundary condition on a bounded domain of $n$ dimensional Euclidean space for a class of nonlinear Ginzburg-Landau-Schrödinger evolution equation is continued. New simple sufficient conditions have been obtained for a wide class of initial data under which collapse happens for the given new values of parameters.

\section{Introduction}

In the present paper, the investigation of the blow-up of solutions of the problem for the first mixed-value problem of the Ginzburg-Landau-Schrödinger equation is continued.

Let $\Omega \subset \mathbb{R}^{n}$ be a bounded domain with a smooth boundary $\partial \Omega$. We consider the following mixed-value problem:

$$
\begin{gathered}
u_{t}=(\alpha+i \beta) \Delta u+f(u)+(\eta+i \mu) u, \quad x \in \Omega, t>0, \\
u(x, 0)=u_{0}(x), \quad x \in \bar{\Omega}, \\
\left.u(x, t)\right|_{\partial \Omega}=0, \quad t \geq 0 .
\end{gathered}
$$

Here $f(u)=(\omega+i \gamma)|u|^{1+\rho},\{\alpha, \beta, \omega, \gamma, \eta, \mu\} \in \mathbb{R}, \rho \in \mathbb{R}_{+}, \alpha^{2}+\beta^{2} \neq 0$, and $\omega^{2}+\gamma^{2} \neq 0$.

We meet (1.1a) in different fields of applied physics, in nonlinear quantum mechanics, and in the theory of propagation of light waves in a nonlinear media (see, e.g., $[4,14]$ ). For $\alpha=0, \eta=0, f(u)=i \gamma|u|^{\rho} u, \gamma \beta>0$, and $\rho n \geq 4$, the question on blow-up solutions of problem (1.1) is considered in the case $\mu=0$ in [6] and in the case $\mu>0$ in [7]; the Cauchy problem for (1.1a) in the case $\mu=0$ is considered in $[2,6,9,11]$, and so forth. In the case $\alpha=0, \beta=1, \eta=0, \mu=0, f(u)=\gamma|u|^{\rho} u, \rho>0, \gamma \neq 0$, papers by P. L. Lions, T. Cazenave, B. Weissler Fred, W. A. Strauss, J. Shatah, T. Kato, F. Merle, M. Tsutsumi, Y. Tsutsumi, H. Nawa, J. Ginibre, G. Velo, and so forth (see the references in $[9,10]$ ) are devoted to the different properties of the solutions of the Cauchy problem for (1.1a). 
The global solvability of problem (1.1) for $\alpha=0, \beta=1, \eta=0, \mu=0, f(u)=|u|^{\rho} u, \rho>0$ is investigated by Lions in [3]; for $\alpha=0, \beta=-1, \eta=0, \mu=0, f(u)=-i v_{1}|u|^{\rho_{1}} u-i v_{2}|u|^{\rho_{2}} u$, $\nu_{2}>0, \nu_{1} \in \mathbb{R}, \rho_{1}>0, \rho_{2}>0$ by Vladimirov in [12], the author in [7], and others; for $\alpha=0, \beta=1, \eta=0, \mu=0, f(u)=\gamma|u|^{2} u, \gamma \neq 0, n=2$ by Brézis and Gallouet in [1]; for $f(u)=|u|^{\rho} u, \rho>0$ in [5]; and so forth.

In [8], the problem on blow-up of solutions of problem (1.1) is considered and in the case $a_{0}=\lambda_{0} \alpha-\eta \neq 0$, where $\lambda_{0}$ is the first eigenvalue of the spectral problem (2.1), sufficient conditions on $u_{0}$ are suggested under which collapse happens for the given values of the parameters of (1.1a). The conditions on $u_{0}$ suggested in [8] are cumbersome. In the present paper, the simpler sufficient conditions on $u_{0}$ are offered under which in any value $a_{0}$ for given values of the parameters of (1.1a), the solutions of the problem (1.1) end with singularity.

The obtained results are stated in Theorems 3.1, 3.2, and 3.3. The proofs of these theorems are based on the Lemma 4.1, which is deduced from the equality for the solutions of the problem (1.1) and nontrivial solutions of the spectral problem (2.1) (Statement 4.2).

\section{Notations}

Let $\lambda_{0}$ be the first eigenvalue and $v_{0}(x)$ the corresponding first eigenfunction of the following problem:

$$
\begin{gathered}
\Delta v+\lambda v=0, \quad x \in \Omega, \\
\left.v\right|_{\partial \Omega}=0 .
\end{gathered}
$$

It is known that $\lambda_{0}>0, v_{0}(x) \in C^{2}(\Omega) \cap C(\bar{\Omega})$, and $v_{0}(x)>0$ for all $x \in \Omega$ (see, e.g., $[13$, page 434]). Without loss of the generality, we will consider that

$$
\int_{\Omega} v_{0}(x) d x=1
$$

Notations. $a_{0}=\lambda_{0} \alpha-\eta, b_{0}=\lambda_{0} \beta-\mu, k_{1}=c_{1} \omega+c_{2} \gamma, k_{2}=c_{1} \gamma-c_{2} \omega$, where $\left\{c_{1}, c_{2}\right\} \in$ $\mathbb{R}, c_{1}^{2}+c_{2}^{2} \neq 0$, for $c_{2}=0$ we are to set $c_{1}=1$, for $c_{1}=0-c_{2}=1 ; \tilde{y}_{0}=c_{1}\left(\operatorname{Re} u_{0}, v_{0}\right)+$ $c_{2}\left(\operatorname{Im} u_{0}, v_{0}\right),(\cdot, \cdot)$ is a scalar product in $L_{2}(\Omega) ;\|\cdot\|$ is a norm in $L_{2}(\Omega),\|\cdot\|_{q}$ is a norm in $L_{q}(\Omega), q \geq 1, \stackrel{\circ}{W_{2}^{1}}(\Omega), W_{2}^{2}(\Omega)$ are the Sobolev spaces, $B(\Omega) \equiv \stackrel{\circ}{W}_{2}^{1}(\Omega) \cap W_{2}^{2}(\Omega) \cap$ $L_{\rho+1}(\Omega)$.

We pass to the statement of the obtained results.

\section{The results}

We formulate the results in the form of the following three theorems.

Theorem 3.1. Let $\lambda_{0}$ be the first eigenvalue, let $v_{0}(x)$ be a corresponding first eigenfunction of problem (2.1), satisfying the norm condition (2.2), and let $b_{0} \neq 0, k_{1} \neq 0$, and $\varphi=$ $\operatorname{sign}\left(k_{1} k_{2} b_{0}\right) \arcsin \left(\left|k_{2}\right| / \sqrt{k_{1}^{2}+k_{2}^{2}}\right)$. Further, let the initial function $u_{0} \in B(\Omega)$ be such that

$$
y_{0}=\operatorname{sign}\left(k_{1}\right) \tilde{y}_{0}
$$


satisfies the condition

$$
y_{0} \geq\left[\frac{\chi}{(1-\sin \varphi)}\right]^{1 / \rho}
$$

here,

$$
\chi= \begin{cases}\frac{\left|b_{0}\right|}{\rho \chi_{0}} & \text { for } a_{0} \leq 0, \\ \left|b_{0}\right| \frac{\exp \left(a_{0} \rho t_{p}\right)}{\rho \chi_{0}} & \text { for } a_{0}>0,\end{cases}
$$

where

$$
\chi_{0}=\frac{\sqrt{\gamma^{2}+\omega^{2}} \sqrt{c_{1}^{2}+c_{2}^{2}}}{\left(\left|c_{1}\right|+\left|c_{2}\right|\right)^{\rho+1}}, \quad t_{\rho}=\frac{\pi / 2-\varphi}{\left|b_{0}\right|}
$$

Then the solution $u(x, t)$ of problem (1.1) from the class $C([0, T], B(\Omega)) \cap C^{1}([0, T]$, $\left.L_{2}(\Omega)\right)$ blows up in a finite time $t_{\max }$, that is, for $t \rightarrow t_{\max }^{-}$,

$$
\begin{aligned}
\|u(\cdot, t)\| & \longrightarrow \infty, & & \|u(\cdot, t)\|_{\rho+1} \\
\|\nabla u(\cdot, t)\| & \longrightarrow \infty, & \|u(\cdot, t)\|_{W_{2}^{2}(\Omega)} & \longrightarrow \infty
\end{aligned}
$$

Moreover, $t_{\max } \leq t_{k} \leq t_{\rho}$, where

$$
t_{k}=\frac{\arcsin \left(\sin \varphi+\chi / y_{0}^{\rho}\right)-\varphi}{\left|b_{0}\right|} .
$$

Theorem 3.2. Let $\lambda_{0}$ be the first eigenvalue, let $v_{0}(x)$ be a corresponding eigenfunction of problem (2.1), satisfying the norm condition (2.2), and let $b_{0} \neq 0, k_{2} \neq 0, \varphi=\arccos \left(\left|k_{2}\right| /\right.$ $\left.\sqrt{k_{1}^{2}+k_{2}^{2}}\right)$, and $\operatorname{sign}\left(k_{1} k_{2} b_{0}\right)=-1$ for $k_{1} \neq 0$. Further, let the initial function $u_{0} \in B(\Omega)$ be such that

$$
y_{0}=-\operatorname{sign}\left(k_{2} b_{0}\right) \tilde{y}_{0}
$$

satisfies the condition

$$
y_{0} \geq\left[\frac{\chi}{(1+\cos \varphi)}\right]^{1 / \rho},
$$

where $\chi$ is determined by formula (3.3) in which $t_{\rho}=(\pi-\varphi) /\left|b_{0}\right|$, and $\chi_{0}$ is given by relation (3.4).

Then the statement of Theorem 3.1 is valid, where

$$
t_{k}=\frac{\arccos \left(\cos \varphi-\chi / y_{0}^{\rho}\right)-\varphi}{\left|b_{0}\right|} .
$$


Theorem 3.3. Let $\lambda_{0}$ be the first eigenvalue and let $v_{0}(x)$ be a corresponding first eigenfunction of problem (2.1), satisfying the norm condition (2.2). Let $b_{0}=0, k_{1} \neq 0, \omega \neq 0$, and $\gamma \neq 0$ (for $\gamma=0, c_{2}=0$ for $\omega=0-c_{1}=0$ has to be taken). Let the initial function $u_{0}(x) \in B(\Omega)$ be such that

$$
y_{0}=\operatorname{sign}\left(k_{1}\right) \tilde{y}_{0}
$$

in the case $a_{0}>0$ satisfies the condition

$$
y_{0}>\left[\frac{a_{0}}{\chi_{0}}\right]^{1 / \rho},
$$

where

$$
\chi_{0}=\frac{\left|k_{1}\right|}{\left(\left|c_{1}\right|+\left|c_{2}\right|\right)^{\rho+1}}
$$

in the case $a_{0} \leq 0$ satisfies the condition $y_{0}>0$.

Then the statement of Theorem 3.1 is valid, where

$$
t_{k}=-\frac{1}{a_{0} \rho} \ln \left(1-\frac{a_{0}}{\chi_{0} y_{0}^{\rho}}\right)
$$

in the case $a_{0} \neq 0$; in the case $a_{0}=0, t_{k}=1 / \rho \chi_{0} y_{0}^{\rho}$.

\section{Outline of the proof}

4.1. Let $u(x, t) \in C\left(\left[0, t_{\max }\right), B(\Omega)\right) \cap C^{1}\left(\left[0, t_{\max }\right), L_{2}(\Omega)\right)$ be the maximal solution of problem $(1.1)$ in the sense that the interval $\left[0, t_{\max }\right)$ is a maximal interval of the existence of the solution for problem (1.1) from the indicated class. Clearly, $t_{\max }$ is either finite or infinite. By proving the above stated theorems, we use the following lemma.

LEMma 4.1. Let $u_{0}(x) \in B(\Omega), u(x, t)$ be a maximal solution of problem (1.1) from the class $C\left(\left[0, t_{\max }\right), B(\Omega)\right) \cap C^{1}\left(\left[0, t_{\max }\right), L_{2}(\Omega)\right)$, let $\lambda_{0}$ be the first eigenvalue, and let $v_{0}(x)$ be a corresponding first eigenfunction of problem (2.1), satisfying the norm condition (2.2). On the interval $\left[0, t_{\max }\right)$, the following functions are defined:

$$
\begin{aligned}
& y_{1}(t)=\operatorname{Re}\left[\left(u, v_{0}\right) \exp (z t)\right], \\
& y_{2}(t)=\operatorname{Im}\left[\left(u, v_{0}\right) \exp (z t)\right],
\end{aligned}
$$

where $z=a_{0}+i b_{0}$.

Then

(1) in the case $b_{0} \neq 0$ and $k_{1} \neq 0$ for the function $y(t)=\operatorname{sign}\left(k_{1}\right)\left(c_{1} y_{1}(t)+c_{2} y_{2}(t)\right)$ with the condition $y_{0}=\operatorname{sign}\left(k_{1}\right) \tilde{y}_{0}>0$ on the interval $\left[0, t^{*}\right)$, where $t^{*}=\min \left(t_{\max }, t_{\rho}\right)$, $t_{\rho}=(\pi / 2-\varphi) /\left|b_{0}\right|, \varphi=\operatorname{sign}\left(k_{1} k_{2} b_{0}\right) \arcsin \left(\left|k_{2}\right| / \sqrt{k_{1}^{2}+k_{2}^{2}}\right)$, the following differential 
inequality is valid:

$$
\frac{d y}{d t} \geq \chi^{*} \cos \left(\left|b_{0}\right| t+\varphi\right) y^{1+\rho}
$$

here,

$$
\chi^{*}= \begin{cases}\chi_{0} & \text { for } a_{0} \leq 0 \\ \chi_{0} \exp \left(-a_{0} \rho t_{\rho}\right) & \text { for } a_{0}>0\end{cases}
$$

and $\chi_{0}$ has been determined in Theorem 3.1 by formula (3.4);

(2) in the case $b_{0} \neq 0, k_{2} \neq 0$, and $\operatorname{sign}\left(k_{1} k_{2} b_{0}\right)=-1$ in the case $k_{1} \neq 0$ for the function $y(t)=-\operatorname{sign}\left(k_{2} b_{0}\right)\left(c_{1} y_{1}(t)+c_{2} y_{2}(t)\right)$ with the condition $y_{0}=-\operatorname{sign}\left(k_{2} b_{0}\right) \tilde{y}_{0}>0$ on the interval $\left[0, t^{*}\right)$, where $t^{*}=\min \left(t_{\max }, t_{\rho}\right), t_{\rho}=(\pi-\varphi) /\left|b_{0}\right|, \varphi=-\operatorname{sign}\left(k_{1} k_{2} b_{0}\right)$ $\arccos \left(\left|k_{2}\right| / \sqrt{k_{1}^{2}+k_{2}^{2}}\right)$, the following differential inequality is valid:

$$
\frac{d y}{d t} \geq \chi^{*} \sin \left(\left|b_{0}\right| t+\varphi\right) y^{1+\rho}
$$

here $\chi^{*}$ is determined by formula (4.3) in which $t_{\rho}=(\pi-\varphi) /\left|b_{0}\right|$;

(3) in the case $b_{0}=0, k_{1} \neq 0, \omega \neq 0$, and $\gamma \neq 0$ for the function $y(t)=\operatorname{sign}\left(k_{1}\right)\left(c_{1} y_{1}(t)+\right.$ $\left.c_{2} y_{2}(t)\right)$ with the condition $y_{0}=\operatorname{sign}\left(k_{1}\right) \tilde{y}_{0}>0$ on the interval $\left[0, t_{\max }\right)$, the following differential inequality is valid:

$$
\frac{d y}{d t} \geq \chi_{0} e^{-a_{0} \rho t} y^{1+\rho}
$$

where $\chi_{0}=\left|k_{1}\right| /\left(\left|c_{1}\right|+\left|c_{2}\right|\right)^{1+\rho}$.

The above-mentioned lemma is proved on the ground of one suggestion. Now, we pass to the statement.

4.2. An auxiliary affirmation (on an integrodifferential identity for the solution $u(x, t)$ of problem (1.1) and solution $(\lambda, v(x))$ of problem (2.1)). Let $u(x, t) \in C\left(\left[0, t_{\max }\right), B(\Omega)\right)$ $\cap C^{1}\left(\left[0, t_{\max }\right), L_{2}(\Omega)\right)$ be the maximal solution of problem $(1.1)$ and let $(\lambda, v(x))$ be any nontrivial solution of problem (2.1). On the interval $\left[0, t_{\max }\right)$, we introduce the following functions:

$$
\begin{gathered}
y_{1}(t)=\frac{1}{2} \int_{\Omega}\left[e^{z t} u(x, t)+e^{\bar{z} t} \bar{u}(x, t)\right] v(x) d x, \\
y_{2}(t)=-\frac{i}{2} \int_{\Omega}\left[e^{z t} u(x, t)-e^{\bar{z} t} \bar{u}(x, t)\right] v(x) d x,
\end{gathered}
$$

where $z=a+i b, \bar{z}=a-i b, a=\lambda \alpha-\eta, b=\lambda \beta-\eta$, and $\bar{u}(x, t)$ is a complexly adjoint function to the $u(x, t)$. 
The following statement is valid.

STATEMENT 4.2. Let $u(x, t)$ be a maximal solution of problem (1.1) from the class $C\left(\left[0, t_{\max }\right)\right.$, $B(\Omega)) \cap C^{1}\left(\left[0, t_{\max }\right), L_{2}(\Omega)\right)$ and let $(\lambda, v(x))$ be any nontrivial solution of problem (2.1). Let $y_{1}(t), y_{2}(t)$ be functions determined on $\left[0, t_{\max }\right)$ by relations (4.6), respectively.

Then for the functions $y_{1}(t), y_{2}(t)$ on the interval $\left[0, t_{\max }\right)$, the following relations are valid:

$$
\begin{aligned}
& \frac{d y_{1}}{d t}=\exp (a t)[\omega \cos (b t)-\gamma \sin (b t)] I_{\rho}(t), \\
& \frac{d y_{2}}{d t}=\exp (a t)[\omega \sin (b t)-\gamma \cos (b t)] I_{\rho}(t),
\end{aligned}
$$

where $I_{\rho}(t)=\int_{\Omega}|u(x, t)|^{1+\rho} v(x) d x$.

Proof. For $d y_{1} / d t$, we have

$$
\frac{d y_{1}}{d t}=R_{1}(t)+R_{2}(t)
$$

where

$$
\begin{aligned}
& R_{1}(t)=\frac{1}{2} \int_{\Omega}\left[z e^{z t} u(x, t)+\bar{z} e^{\bar{z} t} \bar{u}(x, t)\right] v(x) d x \\
& R_{2}(t)=\frac{1}{2} \int_{\Omega}\left[z e^{z t} u_{t}(x, t)+\bar{z} e^{\bar{z} t} \overline{u_{t}}(x, t)\right] v(x) d x .
\end{aligned}
$$

Taking into account (1.1a) in the right-hand side of the $R_{2}$, we get

$$
\begin{aligned}
& R_{2}(t)=\frac{1}{2} \int_{\Omega}\left\{e^{z t}[(\alpha+i \beta) \Delta u+f(u)+(\eta+i \mu) u]\right. \\
&\left.+e^{\bar{z} t}[(\alpha-i \beta) \Delta \bar{u}+\overline{f(u)}+(\eta+i \mu) \bar{u}]\right\} v(x) d x \\
&= \frac{1}{2}\left\{(\alpha+i \beta) e^{z t} \int_{\Omega} \Delta u v d x+(\alpha-i \beta) e^{\bar{z} t} \int_{\Omega} \overline{\Delta u} v d x\right. \\
&+\int_{\Omega}\left[e^{z t} f(u)+e^{\bar{z} t} \overline{f(u)}\right] v(x) d x+(\eta+i \mu) e^{z t} \int_{\Omega} u v d x \\
&\left.+(\eta-i \mu) e^{\bar{z} t} \int_{\Omega} \bar{u} v d x\right\} .
\end{aligned}
$$

Due to the second Green formula, we have

$$
\int_{\Omega} \Delta u v d x=\int_{\Omega} u \Delta v d x=-\lambda \int_{\Omega} u v d x
$$


Hence,

$$
\begin{aligned}
R_{2}(t)=\frac{1}{2}\{ & -\lambda(\alpha+i \beta) e^{z t} \int_{\Omega} u v d x-\lambda(\alpha-i \beta) e^{\bar{z} t} \int_{\Omega} \bar{u} v d x \\
& +\int_{\Omega}\left[e^{z t} f(u)+e^{\bar{z} t} \overline{f(u)}\right] v(x) d x \\
& \left.+(\eta+i \mu) e^{z t} \int_{\Omega} u v d x+(\eta-i \mu) e^{\bar{z} t} \int_{\Omega} \bar{u} v d x\right\} \\
=\frac{1}{2}\{ & -(\lambda \alpha-\eta) e^{z t} \int_{\Omega} u v d x-(\lambda \alpha-\eta) e^{\bar{z} t} \int_{\Omega} \bar{u} v d x \\
& -i(\lambda \beta-\mu) e^{z t} \int_{\Omega} u v d x+i(\lambda \beta-\mu) e^{\bar{z} t} \int_{\Omega} \bar{u} v d x \\
& \left.+\int_{\Omega}\left[e^{z t} f(u)+e^{\bar{z} t} \overline{f(u)}\right] v d x\right\} \\
=\frac{1}{2}\{ & -a e^{z t} \int_{\Omega} u v d x-a e^{\bar{z} t} \int_{\Omega} \bar{u} v d x-i b e^{z t} \int_{\Omega} u v d x+i b e^{\bar{z} t} \int_{\Omega} \bar{u} v d x \\
& \left.+\int_{\Omega}\left[e^{z t} f(u)+e^{\bar{z} t} \bar{f}(u)\right] v d x\right\} \\
=\frac{1}{2}\{ & -(a+i b) e^{z t} \int_{\Omega} u v d x-(a-i b) e^{\bar{z} t} \int_{\Omega} \bar{u} v d x \\
& \left.+\int_{\Omega}\left[e^{z t} f(u)+e^{\bar{z} t} \bar{f}(u)\right] v d x\right\} \\
=- & \frac{1}{2}\left[z e^{z t} \int_{\Omega} u v d x+\bar{z} e^{\bar{z} t} \int_{\Omega} \bar{u} v d x\right]+\int_{\Omega} \frac{e^{z t} f(u)+e^{\bar{z} t} \bar{f}(u)}{2} v d x \\
= & R_{1}(t)+\int_{\Omega} \frac{e^{z t} f(u)+e^{\bar{z} t} \bar{f}(u)}{2} v d x .
\end{aligned}
$$

Finally, for $d y_{1} / d t$, we get the following relation:

$$
\begin{aligned}
\frac{d y_{1}}{d t} & =R_{1}(t)+R_{2}(t)=\int_{\Omega} \frac{e^{z t} f(u)+e^{\bar{z} t} \bar{f}(u)}{2} v d x \\
& =e^{a t}\left\{\frac{e^{i b t}(\omega+i \gamma)+e^{-i b t}(\omega-i \gamma)}{2}\right\} \int_{\Omega}|u|^{1+\rho} v d x \\
& =e^{a t}\left(\omega \frac{e^{i b t}+e^{-i b t}}{2}-\gamma \frac{e^{i b t}-e^{-i b t}}{2 i}\right) I_{\rho}(t) \\
& =e^{a t}[\omega \cos (b t)-\gamma \sin (b t)] I_{\rho}(t),
\end{aligned}
$$

where $I_{\rho}(t)=\int_{\Omega}|u(x, t)|^{1+\rho} \nu d x$. The proof of relation (4.8) is similar to that of relation (4.7) for $y_{1}(t)$. Hence, we omit it here. The proof of the statement is over.

4.3. Proof of Lemma 4.1. Let $u(x, t)$ be the maximal solution of problem (1.1) from the class $C\left(\left[0, t_{\max }\right), B(\Omega)\right) \cap C^{1}\left(\left[0, t_{\max }\right), L_{2}(\Omega)\right)$. Further, let $c_{1}, c_{2}$ be arbitrary real numbers such that $c_{1}^{2}+c_{2}^{2} \neq 0$. Multiplying (4.7) by $c_{1}$, (4.8) by $c_{2}$, and then adding the results, we 
get the following equation:

$$
\frac{d}{d t}\left(c_{1} y_{1}+c_{2} y_{2}\right)=e^{a t}\left(k_{1} \cos b t-k_{2} \sin b t\right) I_{\rho}(t)
$$

4.3.1. Proof of Lemma 4.1(1). Let $b \neq 0$ and $k_{1} \neq 0$. We represent the function $\Phi(t)=$ $k_{1} \cos b t-k_{2} \sin b t$ in the following form:

$$
\begin{aligned}
\Phi(t) & =\left|k_{1}\right| \operatorname{sign}\left(k_{1}\right) \cos (|b| t)-\left|k_{2}\right| \operatorname{sign}\left(k_{2} b\right) \sin (|b| t) \\
& =\operatorname{sign}\left(k_{1}\right)\left[\left|k_{1}\right| \cos (|b| t)-\left|k_{2}\right| \operatorname{sign}\left(k_{1} k_{2} b\right) \sin (|b| t)\right] \\
& =\operatorname{sign}\left(k_{1}\right) \sqrt{k_{1}^{2}+k_{2}^{2}}\left[\frac{\left|k_{1}\right|}{\sqrt{k_{1}^{2}+k_{2}^{2}}} \cos (|b| t)-\frac{\left|k_{2}\right|}{\sqrt{k_{1}^{2}+k_{2}^{2}}} \operatorname{sign}\left(k_{1} k_{2} b\right) \sin (|b| t)\right] .
\end{aligned}
$$

Introducing the notations

$$
\cos \varphi_{0}=\frac{\left|k_{1}\right|}{\sqrt{k_{1}^{2}+k_{2}^{2}}}, \quad \sin \varphi_{0}=\frac{\left|k_{2}\right|}{\sqrt{k_{1}^{2}+k_{2}^{2}}}
$$

for $\Phi(t)$, we have the following expression:

$$
\Phi(t)=\operatorname{sign}\left(k_{1}\right) \sqrt{k_{1}^{2}+k_{2}^{2}} \cos (|b| t+\varphi),
$$

where

$$
\varphi=\operatorname{sign}\left(k_{1} k_{2} b\right) \varphi_{0}, \quad \varphi_{0}=\arcsin \frac{\left|k_{2}\right|}{\sqrt{k_{1}^{2}+k_{2}^{2}}}
$$

Substituting it into (4.15), we get for all $t \in\left[0, t_{\max }\right)$ the following equation:

$$
\frac{d y}{d t}=e^{a t} \sqrt{k_{1}^{2}+k_{2}^{2}} \cos (|b| t+\varphi) I_{\rho}(t)
$$

where $y=\operatorname{sign}\left(k_{1}\right)\left(c_{1} y_{1}+c_{2} y_{2}\right)$. The function $\cos (|b| t+\varphi)$ in the segment $\left[0, t_{\rho}\right]$, where $t_{\rho}=(\pi / 2-\varphi) /|b|$, is nonnegative. The function

$$
I_{\rho}(t)=\int_{\Omega}|u(x, t)|^{1+\rho} v(x) d x
$$

will be positive for all $t \in\left[0, t_{\max }\right)$ if $v(x)=v_{0}(x)$. Therefore, choosing $\lambda=\lambda_{0}$ and $v(x)=$ $v_{0}(x)$, we see obviously that the right-hand side of (4.20) has the positive sign in $t \in$ $\left[0, t^{*}\right)$, where $t^{*}=\min \left(t_{\max }, t_{\rho}\right)$. Suppose that $y_{0}=\operatorname{sign}\left(k_{1}\right) \tilde{y}_{0}>0$. Then from $(4.20)$, we deduce that $y(t)$ in $\left[0, t^{*}\right)$ strictly increases, and hence is strictly positive. For $y(t)$ in 
$\left[0, t^{*}\right)$, we have the following estimate:

$$
\begin{aligned}
y(t) \leq & \left(\left|c_{1}\right|\left|y_{1}\right|+\left|c_{2}\right|\left|y_{2}\right|\right) \\
\leq & \left(\left|c_{1}\right|+\left|c_{2}\right|\right) e^{a_{0} t} \int_{\Omega}|u(x, t)| v_{0}(x) d x \\
= & \left(\left|c_{1}\right|+\left|c_{2}\right|\right) e^{a_{0} t} \int_{\Omega}|u| v_{0}^{1 /(\rho+1)} v_{0}^{\rho /(\rho+1)} d x \quad \text { (by Hölder's inequality for integrals) } \\
\leq & \left(\left|c_{1}\right|+\left|c_{2}\right|\right) e^{a_{0} t}\left(\int_{\Omega}|u(x, t)|^{\rho+1} v_{0}(x) d x\right)^{1 /(\rho+1)} \\
& \times\left(\int_{\Omega} v_{0}(x) d x\right)^{\rho /(\rho+1)} \quad(\text { by the norm condition }(2.2)) \\
= & \left(\left|c_{1}\right|+\left|c_{2}\right|\right) e^{a_{0} t} I_{\rho}^{1 /(\rho+1)}(t) .
\end{aligned}
$$

From this estimate, we deduce for all $t \in\left[0, t^{*}\right)$ the inequality

$$
I_{\rho}(t) \geq \frac{e^{-a_{0}(1+\rho) t}}{\left(\left|c_{1}\right|+\left|c_{2}\right|\right)^{1+\rho}} y^{1+\rho}(t),
$$

due to which, by (4.20) for $y(t)$, we finally obtain the nonlinear differential inequality

$$
\frac{d y}{d t} \geq \frac{e^{-a_{0} \rho t} \sqrt{k_{1}^{2}+k_{2}^{2}}}{\left(\left|c_{1}\right|+\left|c_{2}\right|\right)^{1+\rho}} \cos \left(\left|b_{0}\right| t+\varphi\right) y^{1+\rho}
$$

in which, further taking into account that $e^{-a_{0} \rho t} \geq 1$ in the case $a_{0} \leq 0, e^{-a_{0} \rho t}>e^{-a_{0} \rho t_{\rho}}$ for all $t \in\left[0, t^{*}\right)$ in the case $a_{0}>0$, we get the nonlinear differential inequality

$$
\frac{d y}{d t} \geq \chi^{*} \cos \left(\left|b_{0}\right| t+\varphi\right) y^{1+\rho}
$$

with the initial condition $y_{0}=y(0)=\operatorname{sign}\left(k_{1}\right) \tilde{y}_{0}>0$. Here, $\chi^{*}$ is determined by formula (4.3), and $\chi_{0}$ by (3.4).

The proof of the first part of lemma is over.

4.3.2. Proof of Lemma 4.1(2). Let $b \neq 0$ and $k_{2} \neq 0$. For $\Phi(t)=k_{1} \cos b t-k_{2} \sin b t$, we have

$$
\begin{aligned}
\Phi(t) & =-\operatorname{sign}\left(k_{2} b\right)\left[\left|k_{2}\right| \sin (|b| t)-\left|k_{2}\right| \operatorname{sign}\left(k_{1} k_{2} b\right) \cos (|b| t)\right] \\
& =-\operatorname{sign}\left(k_{2} b\right) \sqrt{k_{1}^{2}+k_{2}^{2}}\left[\frac{\left|k_{2}\right|}{\sqrt{k_{1}^{2}+k_{2}^{2}}} \sin (|b| t)-\frac{\left|k_{1}\right|}{\sqrt{k_{1}^{2}+k_{2}^{2}}} \operatorname{sign}\left(k_{1} k_{2} b\right) \cos (|b| t)\right] .
\end{aligned}
$$

Introducing the notations

$$
\cos \varphi_{0}=\frac{\left|k_{2}\right|}{\sqrt{k_{1}^{2}+k_{2}^{2}}}, \quad \sin \varphi_{0}=\frac{\left|k_{1}\right|}{\sqrt{k_{1}^{2}+k_{2}^{2}}}
$$


finally for $\Phi(t)$, we have the following expression:

$$
\begin{aligned}
\Phi(t) & =-\operatorname{sign}\left(k_{2} b\right) \sqrt{k_{1}^{2}+k_{2}^{2}}\left[\cos \varphi_{0} \sin (|b| t)-\operatorname{sign}\left(k_{1} k_{2} b\right) \sin \varphi_{0} \cos (|b| t)\right] \\
& =-\operatorname{sign}\left(k_{2} b\right) \sqrt{k_{1}^{2}+k_{2}^{2}} \sin (|b| t+\varphi),
\end{aligned}
$$

where

$$
\varphi=\operatorname{sign}\left(k_{1} k_{2} b\right) \varphi_{0}, \quad \varphi_{0}=\arccos \frac{\left|k_{2}\right|}{\sqrt{k_{1}^{2}+k_{2}^{2}}}
$$

Substituting this expression for $\Phi(t)$ into (4.15) for all $t \in\left[0, t_{\max }\right)$, we get the following equation:

$$
\frac{d y}{d t}=e^{a t} \sqrt{k_{1}^{2}+k_{2}^{2}} \sin (|b| t+\varphi) I_{\rho}(t)
$$

where $y=-\operatorname{sign}\left(k_{2} b\right)\left(c_{1} y_{1}+c_{2} y_{2}\right)$. Let $k_{1} \neq 0$ and $\operatorname{sign}\left(k_{1} k_{2} b\right)=-1$. Then $\sin (|b| t+$ $\left.\varphi_{0}\right)$ in the segment $\left[0, t_{\rho}\right]$, where $t_{\rho}=\left(\pi-\varphi_{0}\right) /|b|$, is nonnegative. In addition, $I_{\rho}(t)=$ $\int_{\Omega}|u(x, t)|^{1+\rho} v(x) d x$ will be positive for all $t \in\left[0, t_{\max }\right)$ if we have to take $v(x)=v_{0}(x)$. Hence, under choosing $\lambda=\lambda_{0}$ and $v(x)=v_{0}(x)$ for all $t \in\left[0, t^{*}\right)$, where $t^{*}=\min \left(t_{\max }, t_{\rho}\right)$ by virtue of (4.30), we conclude that $y(t)$ strictly increases; therefore, $y(t) \geq y_{0}$. Further, by analogical considerations, which have been done in the proof of the first part of the lemma, we establish the following inequality:

$$
I_{\rho}(t) \geq \chi_{0} e^{-a_{0}(1+\rho) t} y^{1+\rho}(t)
$$

Taking into account the last estimate for $I_{\rho}(t)$ in (4.30), we get the following nonlinear differential inequality:

$$
\frac{d y}{d t} \geq \chi_{0} e^{-a_{0} \rho t} \sin \left(\left|b_{0}\right| t+\varphi_{0}\right) y^{1+\rho}
$$

with the initial data $y_{0}=-\operatorname{sign}\left(k_{2} b_{0}\right) \tilde{y}_{0}>0$ from which, obviously as in the proof of the first part of the lemma, one has

$$
\frac{d y}{d t} \geq \chi^{*} \sin \left(\left|b_{0}\right| t+\varphi_{0}\right) y^{1+\rho}
$$

where $\chi^{*}$ is determined by formula (4.3) with its $t_{\rho}$.

The proof of the second part of the lemma is over.

4.3.3. Proof of Lemma 4.1(3). Let $\omega \neq 0, \gamma \neq 0,\left\{c_{1}, c_{2}\right\} \in \mathbb{R}, c_{1}^{2}+c_{2}^{2} \neq 0, \lambda=\lambda_{0}, v(x)=$ $v_{0}(x)$, and $b_{0}=\lambda_{0} \beta-\nu=0$. Then from (4.15), we deduce that the following equation is true:

$$
\frac{d y}{d t}=e^{a_{0} t}\left|k_{1}\right| I_{\rho}(t)
$$


where $y=\operatorname{sign}\left(k_{1}\right)\left(c_{1} y_{1}+c_{2} y_{2}\right)$ from which similarly to the proof of the first and second parts of the lemma, obviously one has

$$
\frac{d y}{d t} \geq \chi_{0} e^{-a_{0} \rho t} y^{1+\rho}
$$

where $\chi_{0}=\left|k_{1}\right| /\left(\left|c_{1}\right|+\left|c_{2}\right|\right)^{1+\rho}$ with the initial condition $y_{0}=\operatorname{sign}\left(k_{1}\right) \tilde{y}_{0}>0$. The proof of the third part of the lemma is over.

4.4. Proof of the theorems. We will prove in detail only Theorem 3.1 because the proofs of Theorems 3.2 and 3.3 are similar to that of Theorem 3.1, hence we will omit them here. Let all conditions of Theorem 3.1 be fulfilled; $u(x, t)$ is the maximal solution of problem (1.1). Let $t_{\max }$ be finite. In this case, we show that $t_{\max } \leq t_{k}$. We will prove this claim by contradiction, that is, we assume that $t_{\max }>t_{k}$. By virtue of the first part of the lemma for the function $y=\operatorname{sign}\left(k_{1}\right)\left(c_{1} y_{1}+c_{2} y_{2}\right)$ under conditions of Theorem 3.1, the following nonlinear differential inequality is fulfilled for $t \in\left[0, t^{*}\right), t^{*}=\min \left(t_{\max }, t_{\rho}\right)$, where $t_{\rho}$ is determined in Theorem 3.1:

$$
\frac{d y}{d t} \geq \chi^{*} \cos \left(\left|b_{0}\right| t+\varphi\right) y^{1+\rho}
$$

with initial data $y_{0}=\operatorname{sign}\left(k_{1}\right) \tilde{y}_{0}>0$ from which, after separation of the variables by the well-known procedure, we conclude that for $y(t)$, the following lower bound estimate is valid:

$$
y(t) \geq \frac{y_{0}}{F(t)^{1 / \rho}}
$$

where $F(t)=1-y_{0} \rho \chi^{*}\left[\sin \left(\left|b_{0}\right| t+\varphi\right)-\sin \varphi\right] /\left|b_{0}\right|$.

To finish the proof of Theorem 3.1, one has to estimate the norms $\|u(\cdot, t)\|$, $\|u(\cdot, t)\|_{1+\rho},\|\nabla u(\cdot, t)\|$, and $\|u(\cdot, t)\|_{W_{2}^{2}(\Omega)}$ from below by $y(t)$ in $t \in\left[0, t^{*}\right)$. For $y(t)$ on the base of the definitions of $y_{1}(t)$ and $y_{2}(t)$ by the Hölder inequality for integrals, we have the following estimate:

$$
\begin{aligned}
y(t) & \leq e^{a_{0} t}\left(\left|c_{1}\right|+\left|c_{2}\right|\right) \int_{\Omega}|u(x, t)| v_{0}(x) d x \\
& \leq e^{a_{0} t}\left(\left|c_{1}\right|+\left|c_{2}\right|\right)\|u(\cdot, t)\|_{1+\delta}|| v_{0} \|_{(1+\delta) / \delta}
\end{aligned}
$$

for any admissible positive $\delta$. From this inequality for the norm $\|u(\cdot, t)\|_{1+\delta}$, we obtain the lower estimate

$$
\|u(\cdot, t)\|_{1+\delta} \geq \frac{e^{-a_{0} t}}{\left(\left|c_{1}\right|+\left|c_{2}\right|\right)\left\|v_{0}\right\|_{(1+\delta) / \delta}} y(t),
$$

from which, by virtue of the Poincaré inequality $\|\nabla u(\cdot, t)\| \geq$ const $\|u(\cdot, t)\|$, we have

$$
\|\nabla u(\cdot, t)\| \geq c e^{-a_{0} t} y(t)
$$


(here and below, by $c$ we will denote different constants which are independent of $t$ and different norms of $u(x, t)$ ), and by virtue of Sobolev's inequality

$$
\|u(\cdot, t)\|_{W_{2}^{2}(\Omega)} \geq \text { const }\|u(\cdot, t)\|,
$$

we have

$$
\|u(\cdot, t)\|_{W_{2}^{2}(\Omega)} \geq c e^{-a_{0} t} y(t) .
$$

From these estimates and (4.37) for $y(t)$ in $t \in\left[0, t^{*}\right)$ for the norms $\|u(\cdot, t)\|,\|u(\cdot, t)\|_{1+\rho}$, $\|\nabla u(\cdot, t)\|$, and $\|u(\cdot, t)\|_{W_{2}^{2}(\Omega)}$, we get the following lower estimates:

$$
\begin{aligned}
& \|u(\cdot, t)\| \geq \frac{c e^{-a_{0} \rho t}}{R(t)}, \quad\|u(\cdot, t)\|_{1+\rho} \geq \frac{c e^{-a_{0} \rho t}}{R(t)}, \\
& \|\nabla u(\cdot, t)\| \geq \frac{c e^{-a_{0} \rho t}}{R(t)}, \quad\|u(\cdot, t)\|_{W_{2}^{2}(\Omega)} \geq \frac{c e^{-a_{0} \rho t}}{R(t)},
\end{aligned}
$$

where $R(t)=F^{1 / \rho}(t)$.

We pay attention to these estimates. Function $F(t)$ is defined and continues for all $t \geq 0$. At the point $t=0$, it has the value $F(0)=1$. We calculate its value at the point $t=t_{\rho}$. We have $F\left(t_{\rho}\right)=1-y_{0}^{\rho}(1-\sin \varphi) / \chi$, where $\chi$ has been determined in Theorem 3.1 by formula (3.3). By virtue of the condition put on $y_{0}, y_{0} \geq[\chi /(1-\sin \varphi)]^{1 / \rho}$, it follows that $F\left(t_{\rho}\right) \leq 0$. Hence, the function $F(t)$ in the segment $\left[0, t_{\rho}\right]$ decreasingly intersects it at the unique point $t_{k} \in\left(0, t_{\rho}\right]$, which is the unique root in $\left(0, t_{\rho}\right]$ of the trigonometric equation

$$
\sin \left(\left|b_{0}\right| t+\varphi\right)=\sin \varphi+\frac{\chi}{y_{0}^{\rho}}
$$

and is expressed by the formula

$$
t_{k}=\frac{\arcsin \left(\sin \varphi+\chi / y_{0}^{\rho}\right)-\varphi}{\left|b_{0}\right|} .
$$

It is clear that $F(t)<0$ for $t>t_{k}$, so $R(t)$ has been determined only in the segment $\left[0, t_{k}\right]$. By our assumption, the solution $u(x, t)$ of problem (1.1) from the class $C\left(\left[0, t_{\max }\right), B(\Omega)\right)$ $\cap C^{1}\left(\left[0, t_{\max }\right), L_{2}(\Omega)\right)$ exists in $\left[0, t_{k}\right] \cup\left[t_{k}, t^{*}\right)$. Therefore, owing to our assumption in $\left[0, t_{k}\right], y(t),\|u(\cdot, t)\|,\|u(\cdot, t)\|_{1+\rho},\|\nabla u(\cdot, t)\|$, and $\|u(\cdot, t)\|_{W_{2}^{2}(\Omega)}$ are determined. But from estimates (4.43), it follows that $\|u(\cdot, t)\| \rightarrow \infty,\|u(\cdot, t)\|_{1+\rho} \rightarrow \infty,\|\nabla u(\cdot, t)\| \rightarrow \infty$, and $\|u(\cdot, t)\|_{W_{2}^{2}(\Omega)} \rightarrow \infty$ as $t \rightarrow t_{k}$. We obtained the contradiction as consequences of it. One has to state that $t_{\max } \leq t_{k}$, and therefore, the claim of Theorem 3.1 is true. The proof of Theorem 3.1 is over.

\section{Acknowledgments}

I would like to express my deep appreciation to Prof. M. V. Vladimirov for his encouragement and support, Prof. H. Brézis for his benevolent attitude in regard to my paper and useful advice concerning the publication of it, and also Prof. Y. Mamedov for being considerate to my work. 


\section{References}

[1] H. Brézis and T. Gallouet, Nonlinear Schrödinger evolution equations, Nonlinear Anal. 4 (1980), no. 4, 677-681.

[2] O. I. Kudrjašov, Singularities of the solutions of nonlinear equations of Ginzburg-Landau type, Sibirsk. Mat. Ž. 16 (1975), no. 4, 866-868.

[3] J. L. Lions, Some Methods for the Solution of Non-Linear Boundary Value Problems, Nauka, Moscow, 1972.

[4] V. I. Lugovoy and A. M. Prokhorov, Theory of propagation of powerful lazer radiation, Uspekhi Fiz. Nauk 111 (1973), no. 2, 203-247.

[5] Š. M. Nasibov, A nonlinear equation of Schrödinger type, Differencial'nye Uravnenija 16 (1980), no. 4, 660-670 (Russian).

[6] Sh. M. Nasibov, Stability, breakdown, damping and self-channeling of solutions of a nonlinear Schrödinger equation, Dokl. Akad. Nauk SSSR 285 (1985), no. 4, 807-811 (Russian).

[7] A nonlinear Schrödinger equation with a dissipative term, Dokl. Akad. Nauk SSSR 304 (1989), no. 2, 285-289.

[8] On a class of nonlinear Ginzburg-Landau-Schrödinger evolution equations, Dokl. Akad. Nauk 376 (2001), no. 5, 605-607 (Russian).

[9] H. Nawa, Asymptotic and limiting profiles of blowup solutions of the nonlinear Schrödinger equation with critical power, Comm. Pure Appl. Math. 52 (1999), no. 2, 193-270.

[10] M. Ohta, Stability of solitary waves for coupled nonlinear Schrödinger equations, Nonlinear Anal. 26 (1996), no. 5, 933-939.

[11] A. B. Shabat, On Cauchy problem for Ginzburg-Landau equations, Dinamika Sploshn. Sredy (1969), no. 1, 180-194 (Russian).

[12] M. V. Vladimirov, Solvability of a mixed problem for the nonlinear Schrödinger equation, Mat. Sb. (N.S.) 130(172) (1986), no. 4, 520-536 (Russian).

[13] V. S. Vladimirov, The Equations of Mathematical Physics, Nauka, Moscow, 1981.

[14] V. E. Zakharov and A. B. Shabat, Exact theory of two-dimensional self-focusing and onedimensional self-modulation of waves in nonlinear media, Ž. Èksper. Teoret. Fiz. 61 (1971), no. 1, 118-134 (Russian).

Sh. M. Nasibov: Institute for Applied Mathematics, Baku State University, 23 Z.Khalilov Street, 370148 Baku, Azerbaijan

E-mail address: nasibov_sharif@bsu.az 


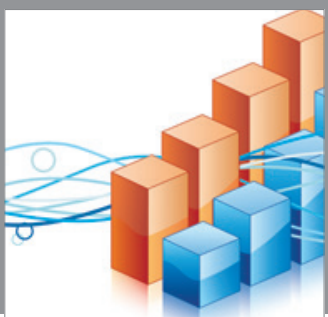

Advances in

Operations Research

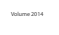

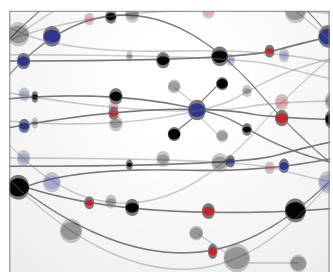

\section{The Scientific} World Journal
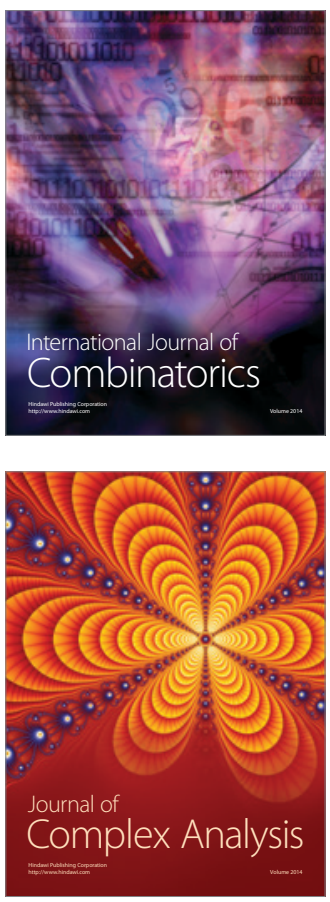

International Journal of

Mathematics and

Mathematical

Sciences
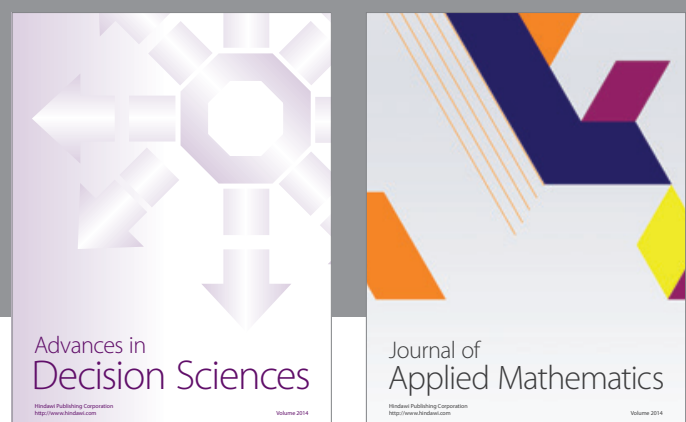

Journal of

Applied Mathematics
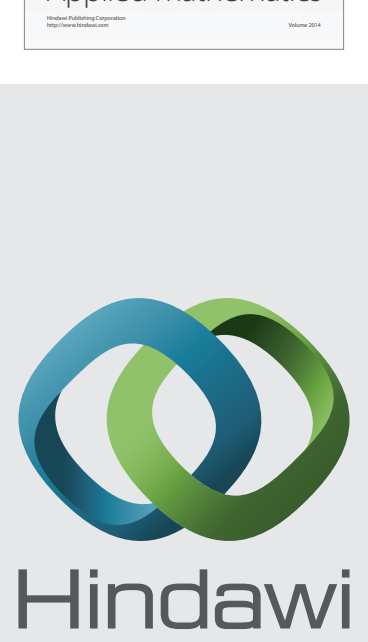

Submit your manuscripts at http://www.hindawi.com
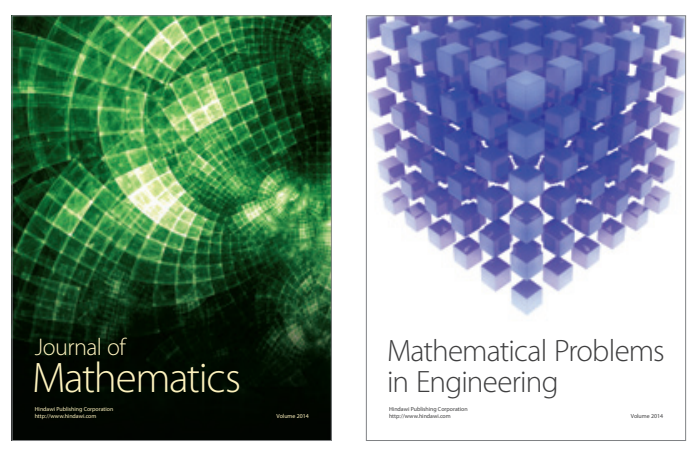

Mathematical Problems in Engineering
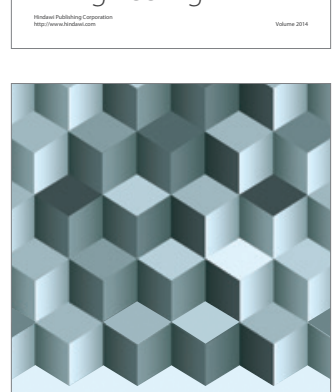

Journal of

Function Spaces
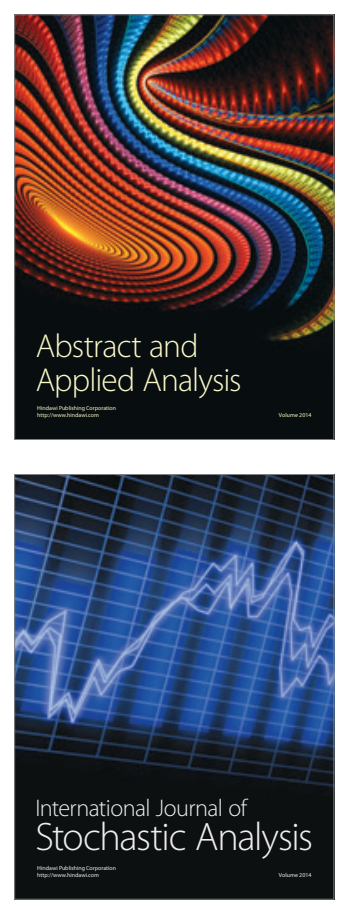

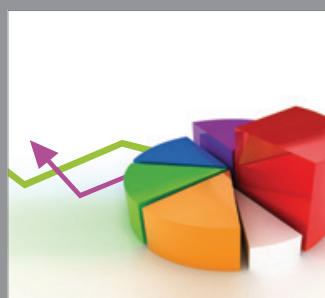

ournal of

Probability and Statistics

Promensencen
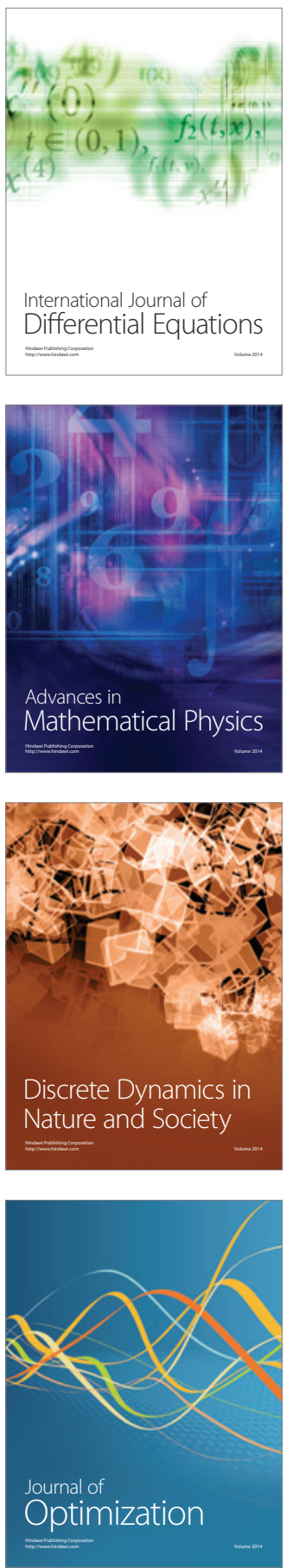